\title{
The File-Card-Browser View for Breast DCE-MRI Data
}

\author{
Sylvia Glaßer ${ }^{1}$, Kathrin Scheil ${ }^{1}$, Uta Preim ${ }^{2}$, Bernhard Preim ${ }^{1}$ \\ ${ }^{1}$ Department of Simulation and Graphics, University of Magdeburg \\ ${ }^{2}$ Department of Radiology, University Hospital Magdeburg \\ glasser@isg.cs.uni-magdeburg.de
}

\begin{abstract}
Dynamic contrast-enhanced magnetic resonance imaging of the breast is acquired for the detection of breast cancer. To rate a tumor to be benign or malignant, radiologists evaluate the tumor's morphology and its enhancement kinetics. We present a new multi planar reformatting (MPR) view, the File-Card-Browser View, to improve and complete the standard axial slice-based evaluation. We tested our technique with a tumor set containing 20 cases and present first results.
\end{abstract}

\section{Introduction}

In 2008, breast cancer was the most common incident cancer and cause of cancerrelated death in women [1]. During the last decades, several image acquisition techniques have been employed for breast cancer diagnosis. Dynamic contrastenhanced magnetic resonance imaging (DCE-MRI) is currently the most sensitive modality for the detection of invasive breast cancer [2]. A great advantage of DCE-MRI is the ability to reveal small tumors that could not be detected with X-ray mammography. For the accurate assessment of breast cancer in DCEMRI, no standardized evaluation guidelines but two main evaluation criteria exist: the morphology of the tumor and the tumor's enhancement kinetics [2].

With focus on the first criterion, we present a new multi planar reformatting (MPR) view for an improved evaluation of a tumor's morphology. As depicted in Fig. 1(a), benign tumors are mostly characterized by round boundaries whereas a stellated morphology indicates a malignant tumor. In clinical practice, radiologists employ 2D slice-based views for morphology evaluation of tumors in DCE-MRI data. However, stellated parts of a tumor's boundary may not be recognizable in the current slice-based view, e.g. the axial slice view, see Fig. 1(b). Hence, an additional view could reduce misinterpretations.

\section{Materials and Methods}

For the evaluation of breast cancer in DCE-MRI datasets, different visualization and evaluation methods exist. In [4], basic techniques for the visualization of breast cancer in DCE-MRI are presented. Englmeier et al. [5] employ a morphofunctional 3D visualization and the MammaExplorer [6] includes interaction, 
segmentation and visualization techniques for breast cancer. Beyond the standard axial slice-based 2D view, sagittal and transversal views can be evaluated in addition. For special diagnostic tasks, such as the diagnosis of vascular diseases, adapted 2D views like MPR as well as the curved multi planar reconstruction [7] were developed.

We present a semi-automatic generation of MPR views for the evaluation of a tumor's morphology. Similar to a conventional file card browser, our FileCard-Browser (FCB) View provides 2D views, which can be rotated around the tumor. The creation of the MPR views comprises four steps that are explained in the following.

We applied our technique to 16 breast DCE-MRI datasets, containing 20 tumors. The datasets were acquired with a $1.0 \mathrm{~T}$ open MRI scanner and exhibit the parameters: in-plane resolution $\approx 0.67 \times 0.67 \mathrm{~mm}^{2}$, matrix $\approx 528 \times 528$, number of slices $\approx 100$, slice gap $=1.5 \mathrm{~mm}$, number of acquisitions $=5-6$ and total acquisition time $\approx 400 \mathrm{sec}$. Since DCE-MRI data exhibit motion artifacts mainly due to thorax expansion through breathing and patient's movement, motion correction was carried out with MeVisLab (www.mevislab.de), employing the elastic registration method developed by Rueckert et al. [8]. Afterwards, isotropic voxel size is achieved by resampling with the Lanzcos Filter. Finally, the relative enhancement $(R E)$ of a tumor, ie. the percent aged signal intensity increase, is calculated [9]

$$
R E=\frac{\left(S I_{c}-S I\right)}{S I} \times 100
$$

where $S I$ is the pre-contrast and $S I_{c}$ is the post-contrast signal intensity.

With a one-click user interaction, a seed point is placed inside the tumor tissue. Next, region growing is applied to extract all connected tumor voxels that exhibit at least $50 \% R E$ at the first time step after the early post contrast phase (Fig. 2a,b). In case a supplying vessel or parts of the surrounding tissue also exhibit contrast agent enhancement, the user can crop the extracted mask with primitive clipping.

The center $c$ is approximated as mean position of the masked voxels.

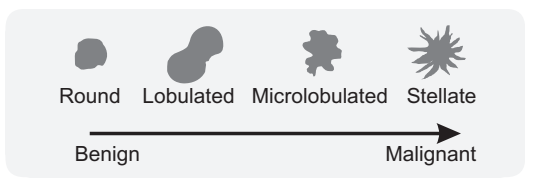

(a)

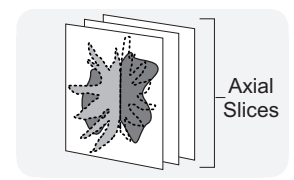

(b)

Fig. 1. In (a), morphological shape types of breast cancer are depicted (adapted from [3]). In (b), a tumor with a stellated boundary (light gray), perpendicular to the viewing plane (e.g. axial viewing slices), is presented. If the tumor's boundary at the current viewing slice (dark gray) exhibit more lobulated parts instead of stellated parts, the boundary evaluation could be error-prone. 
With the PCA, the first and second principal components $p c_{1}$ and $p c_{2}$ of all masked tumor voxels are extracted. Thus, $p c_{1}$ and $p c_{2}$ correspond to the two main directions of the tumor. Next, the MPR plane is generated based on the plane defined by $p c_{1}$ and $p c_{2}$ (Fig. 2c). For the generation of the FCB View, we chose the first vector $p c_{1}$ as rotation axis and the MPR plane and its rotation is adjusted to the tumor's main directions. The user can rotate around $180^{\circ}$ for a complete overview of the tumor's boundary (Fig. 2d).

\section{Results}

We tested the FCB View in two ways. First, we analyzed the tumor masks' extents by means of the PCA results and second, an informal, qualitative evaluation was conducted.

For all 20 tumors, we calculated the angles between $p c_{1}$ and $p c_{2}$ with the two unit vectors $e_{1}=(1,0,0)^{T}$ and $e_{2}=(0,1,0)^{T}$, since the standard slicebased view employs $e_{1}$ and $e_{2}$ as plane vectors. Next, $p c_{1}$ is compared to $e_{1}$, if the angle $\angle\left(p c_{1}, e_{1}\right)$ is smaller than the angle $\angle\left(p c_{1}, e_{2}\right)$. Then, the remaining unit vector is compared to $p c_{2}$. The comparison yields two angles, denoting the angles between $p c_{1}$ and $p c_{2}$ and their corresponding unit vectors. As a result, we obtained the average angle $w_{\mu}$

$$
w_{\mu}=49.54^{\circ}
$$

The amount of $w_{\mu}$ indicates a strong mean deviation of the tumor's main directions to the vectors $e_{1}$ and $e_{2}$, which is a motivation for our method. In the qualitative study, we created the FCB View for all tumors. In Fig. 3 the results are provided by showing four interesting examples. The first case presents a small, round benign tumor. As it is depicted, the FCB View reveals a round boundary, too. In the second case, a more stellated tumor can be observed in the conventional slice-based view. With our technique, two separate parts of the tumor become visible and thus, the tumor's morphology can be better understood.

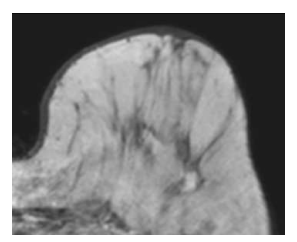

(a)

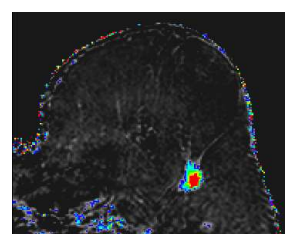

(b)

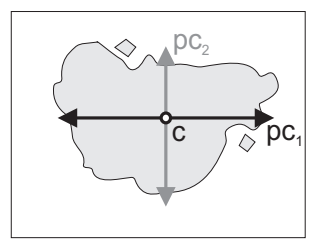

(c)

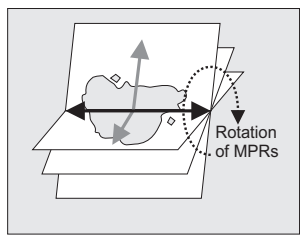

(d)

Fig. 2. Creation of the MPR views. In (a), an axial slice of the source image is depicted. In (b) voxels of the tumor with $R E$ values $>50 \%$ are color coded. By applying this threshold of $50 \%$, the user can select the tumor with one click in most cases. For the presented example, the tumor scheme is provided in (c). The first two prinicipal components $p c_{1}$ and $p c_{2}$ are depicted. The MPR plane is generated with the vectors $p c_{1}$ and $p c_{2}$, and $p c_{1}$ is employed as rotation axis (d). 
The example in the third case serves as representation for different tumor parts with different enhancing characteristics. Although both views present a tumor with an irregular boundary, our technique reveals two similarly perfused regions (visualized with similar color coded contrast enhancement kinetics), whereas the conventional view could not show these spatial connected and similarly perfused parts. Similarly perfused regions of a tumor are necessary for evaluating the tumor's heterogeneity as well as further diagnosis like core needle biopsy. In 2 of 20 cases, the center $c$ was not optimal located due to a very strong stellated and irregular morphology. In these cases, the FCB View could not provide the desired improved overview. However, such irregularly shaped tumors can be already evaluated in the conventional slice-based view and do not need further evaluation with an additional MPR view.

As a result, the FCB View is adapted to each tumor, taking the tumor's spatial extent into account. Furthermore, it provides additional information about the tumor's morphology and boundary. Thus, different parts, which are not
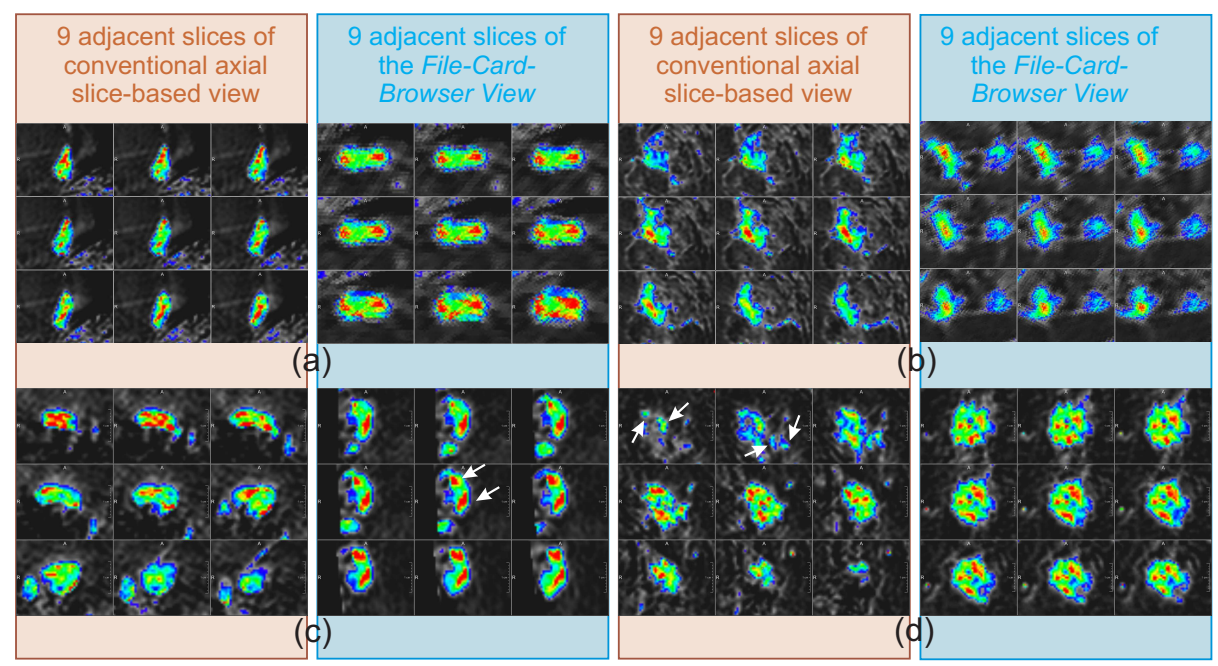

Fig. 3. Four representative examples of our tumor set. On the left, adjacent slices in the conventional slice-based view are presented. On the right, adjacent slices of the same tumor with the FCB View are shown (due to the generation, the slices of the different techniques are not identical). For mapping of $R E$, color coding is applied. In (a), a small round benign tumor is depicted. This boundary shape can be observed in both views. In (b), a more stellated tumor can be observed on the left. With our technique, two separate spatially non-connected parts of the tumor become visible. The example in (c) serves as representation for different tumor parts with different enhancing characteristics. The FCB View reveals two similarly perfused regions (see red regions), whereas the conventional view could not show these different enhancing regions. In (d), the improved boundary evaluation is illustrated. On the left, some parts of the tumor seem to be suspicious (see arrows). On the right, the FCB View reveals an almost round boundary. 
spatially connected on the one hand or exhibit different contrast agent accumulations on the other hand, could be identified. Our method aims at additional improvement and completion of the conventional axial slice-based view instead of substitution of this clinical evaluation method.

\section{Discussion}

The presented FCB View creates a new MPR plane that is adapted to the tumor's extent. The presented comparison and examples illustrated the main advantages of this method: the improved boundary evaluation as well as the identification of spatial connected and similarly perfused regions. Whereas the first one allows for a better evaluation of the tumor's morphology, the second one is important for further treatment planning. For future work, a big clinical user study should be carried out to assess, whether this method is reliable. Furthermore, the presented technique should be extended to different contrast agents enhancement attributes like washout dynamics for further investigation of spatial connected and similarly perfused tissue parts.

Acknowledgement. This work was supported by the DFG (Priority Program 1335, grant no. PR660/7-1). We thank Fraunhofer MEVIS for providing advanced MeVisLab features.

\section{References}

1. World Cancer Report. International Agency for Research on Cancer; 2008.

2. Kuhl CK. The current status of breast MR imaging: part I. Radiology. 2007;244(2):356-78.

3. Mavroforakis M, Georgiou H, Dimitropoulos N, et al. Significance analysis of qualitative mammographic features, using linear classifiers, neural networks and support vector machines. Eur Radiol. 2005;54:80-9.

4. Behrens U, Teubner J, Evertsz C, et al. Computer-assisted dynamic evaluation of contrast-enhanced-MRI. Proc CARS. 1996; p. 362-7.

5. Englmeier KH, Hellwig G, Griebel J, et al. Morpho-functional visualization of dynamic MR-mammography. Stud Health Technol Inform. 2004;107(2):838-41.

6. Coto E, Grimm S, Bruckner S, et al. MammoExplorer: An advanced CAD application for breast DCE-MRI. In: Proc VMV; 2005. p. 91-8.

7. Kanitsar A, Fleischmann D, Wegenkittl R, et al. CPR: curved planar reformation. Proc IEEE Vis. 2002; p. 37-44.

8. D Rueckert, L Sonoda, C Hayes and et al . Nonrigid registration using freeform deformations: Application to breast MR images. IEEE Trans Med Imaging. $1999 ; 18(8): 712-21$.

9. Kuhl CK, Mielcareck P, Klaschik S, et al. Dynamic breast MR imaging: are signal intensity time course data useful for differential diagnosis of enhancing lesions? Radiology. 1999;211(1):101-10. 